\title{
Normative Study of the Literacy Independent Cognitive Assessment in Illiterate and Literate Elderly Koreans
}

\author{
Seung-Gul Kang ${ }^{1 *}$, Seong-Jin Cho ${ }^{1 *}$, Seung-Ho Ryu², Seong Hye Choi ${ }^{3}$, Seol-Heui Han ${ }^{4}$, \\ Yong S. Shim ${ }^{5}$, Kwang-Pil Ko ${ }^{6}$, Jee H. Jeong ${ }^{7}$, Jun-Young Lee ${ }^{8 凶}$, and Dong Woo Lee ${ }^{9 凶}$ \\ ${ }^{1}$ Department of Psychiatry, Gil Medical Center, Gachon University School of Medicine, Incheon, Republic of Korea \\ ${ }^{2}$ Department of Psychiatry, Konkuk University Hospital, Seoul, Republic of Korea \\ ${ }^{3}$ Department of Neurology, Inha University School of Medicine, Incheon, Republic of Korea \\ ${ }^{4}$ Department of Neurology, Konkuk University School of Medicine, Seoul, Republic of Korea \\ ${ }^{5}$ Department of Neurology, Bucheon St. Mary's Hospital, The Catholic University of Korea School of Medicine, Bucheon, Republic of Korea \\ ${ }^{6}$ Department of Preventive Medicine, Gachon University School of Medicine, Incheon, Republic of Korea \\ ${ }^{7}$ Department of Neurology, Ewha Womans University School of Medicine, Seoul, Republic of Korea \\ ${ }^{8}$ Department of Psychiatry and Behavioral Science, Seoul National University College of Medicine, SMG-SNU Boramae Medical Center, Seoul, \\ Republic of Korea \\ ${ }^{9}$ Department of Psychiatry, Inje University Sanggye Paik Hospital, Seoul, Republic of Korea
}

Objective The aim of this study was to provide normative data on the Literacy Independent Cognitive Assessment (LICA) and to explore the effects of age, education/literacy, and gender on the performance of this test.

Methods Eight hundred and eighty-eight healthy elderly subjects, including 164 healthy illiterate subjects, participated in this study. None of the participants had serious medical, psychiatric, or neurological disorders including dementia. Bivariate linear regression analyses were performed to examine the effects of age, education/literacy, and sex on the score in each of the LICA cognitive tests. The normative scores for each age and education/literacy groups are presented.

Results Bivariate linear regression analyses revealed that total score and all cognitive tests of the LICA were significantly influenced by both age and education/literacy. Younger and more-educated subjects outperformed older and illiterate or less-educated subjects, respectively, in all of the tests. The normative scores of LICA total score and subset score were presented according to age (60-64, 65-69, $70-74,75-80$, and $\geq 80$ years) and educational levels (illiterate, and $0-3,4-6$, and $\geq 7$ years of education).

Conclusion These results on demographic variables suggest that age and education should be taken into account when attempting to accurately interpret the results of the LICA cognitive subtests. These normative data will be useful for clinical interpretations of the LICA neuropsychological battery in illiterate and literate elderly Koreans. Similar normative studies and validations of the LICA involving different ethnic groups will help to enhance the dementia diagnosis of illiterate people of different ethnicities.

Psychiatry Investig 2015;12(3):305-315

Key Words LICA, Normative study, Age, Education, Literacy.

\section{INTRODUCTION}

Developments in medical science have led to continuing increases in the number of elderly worldwide, with the popu- lation aging faster in Korea than in any other country. ${ }^{1}$ The proportion of the Korean population aged $\geq 65$ years has reached $12.2 \%$, and is expected to reach $38 \%$ by the year $2050 .^{2}$ Although the prevalence of dementia has drastically

\footnotetext{
Received: July 31, 2014 Revised: September 19, 2014 Accepted: September 23, 2014 Available online: May 21, 2015

$\bowtie$ Correspondence: Dong Woo Lee, MD, PhD

Department of Psychiatry, Inje University Sanggye Paik Hospital, 1342 Dongil-ro, Nowon-gu, Seoul 139-707, Republic of Korea

Tel: +82-2-950-1990, Fax: +82-2-950-8069, E-mail: dwlee@paik.ac.kr

$\triangle$ Correspondence: Jun-Young Lee, MD, PhD

Department of Psychiatry and Behavioral Science, Seoul National University College of Medicine, SMG-SNU Boramae Medical Center, 20 Boramae-ro 5-gil, Seoul 156-707, Republic of Korea

Tel: +82-2-870-2462, Fax: +82-2-870-3866, E-mail: benji@snu.ac.kr

*These authors contributed equally to this work.

(a) This is an Open Access article distributed under the terms of the Creative Commons Attribution Non-Commercial License (http://creativecommons.org/licenses/by-nc/3.0) which permits unrestricted non-commercial use, distribution, and reproduction in any medium, provided the original work is properly cited.
} 
increased in parallel with the aging of the overall population, there is as yet no innovative treatment for the condition, and so prompt detection and diagnosis of dementia is very important.

In general, the performance in neuropsychological tests is influenced by educational and cultural factors. ${ }^{3}$ Illiterate people have difficulty in responding to tests that require reading and writing skills or copying complex figures, even though they may have a normal level of cognitive function. Thus, the application rate of neuropsychological tests and accuracy of the findings are both quite low among elderly illiterate subjects and elderly subjects with a low educational level. This finding is particularly pertinent since the worldwide illiteracy rate of adults aged 15 years and older, which was as high as $16 \%$ in 2009 , is expected to be around $15 \%$ by $2015 .{ }^{4,5}$ In South Korea, the illiteracy rate was relatively low in 2008 , at $5.4 \% ;{ }^{6}$ however, the illiteracy and functional-illiteracy rate among those aged in their 70s was reported to be as high as $20.2 \%$ in the same year. ${ }^{7}$ One study performed in a rural agricultural area found that the illiteracy rate was $26.4 \%$ and was associated with a higher risk of Alzheimer's dementia. ${ }^{8}$

The current tests for screening and diagnosing dementia in Korea are simple translation of the Western version, which do not take into consideration the presence of illiteracy in the elderly population. Accordingly, any elderly subject with little or no education but without dementia is likely to have difficulties in properly responding to some of questions of these tests. Therefore, accurate identification and diagnosis of dementia among illiterate elderly subjects is problematic when using these tests. The subjective screening tests for dementia have been developed in an attempt to resolve such inaccuracies, such as the Informant Questionnaire on Cognitive Decline in the Elderly (IQCODE), which has been applied more widely to illiterate and less-educated subjects than has the
Mini-mental State Examination (MMSE). ${ }^{9,10}$ However, since the IQCODE is not a neuropsychological test, it cannot measure the cognitive function of each cognitive domain. Therefore, we have developed a neuropsychological test battery called the Literacy Independent Cognitive Assessment (LICA), which can be used effectively to test illiterate and less-educated Korean subjects, and have subjected it to a validation study. ${ }^{11}$ The authors have also recently developed a short form of this tool and reported on its reliability and validity. ${ }^{12}$ Both the LICA and its short form were confirmed as valid and reliable instruments for diagnosing dementia in either illiterate or literate elderly subjects, and were shown to have good sensitivity and specificity. ${ }^{11,12}$

The aim of this study was to present the normative information for this tool, which is useful for a clinical interpretation of the LICA neuropsychological battery in illiterate and literate elderly Koreans.

\section{METHODS}

\section{Participants}

Eight hundred and eighty-eight healthy elderly subjects (354 males and 534 females; aged 60-90 years) participated in this study, including 164 (18.5\%) healthy illiterate participants (Table 1). Illiteracy was determined using the following procedure: All subjects were asked to read aloud two sentences: "Young-Hee was thirsty due to physical exercise. She opened a refrigerator." They were then asked to write about what would happen next. A subject who could read the sentences and write an appropriate response was determined to be literate. Subjects who were illiterate due to learning and developmental difficulties were excluded from this study.

All of the participants were at least 60 years old and had no history of significant or suggestive decline in their cognitive

Table 1. Demographic characteristics of the participants

\begin{tabular}{lccc}
\hline \multicolumn{1}{c}{ Variables } & Male $(\mathrm{N}=354)$ & Female $(\mathrm{N}=534)$ & Total $(\mathrm{N}=888)$ \\
\hline Age (years) & $72.8 \pm 6.3^{*}$ & $72.1 \pm 6.8$ & $72.4 \pm 6.6$ \\
$60-64$ & $25(7.1 \%)^{\dagger}$ & $66(12.4 \%)$ & $91(10.2 \%)$ \\
$65-69$ & $93(26.3 \%)$ & $136(25.5 \%)$ & $229(25.8 \%)$ \\
$70-74$ & $106(29.9 \%)$ & $138(25.8 \%)$ & $244(27.5 \%)$ \\
$75-79$ & $72(20.3 \%)$ & $110(20.6 \%)$ & $182(20.5 \%)$ \\
$80-$ & $58(16.4 \%)$ & $84(15.7 \%)$ & $142(16.0 \%)$ \\
Illiteracy and education (years) & $8.4 \pm 5.1^{\ddagger}$ & $4.5 \pm 4.3$ & $6.0 \pm 5.0$ \\
Illiteracy & $25(7.1 \%)$ & $139(26.0 \%)$ & $164(18.5 \%)$ \\
$1-3$ & $44(12.4 \%)$ & $123(23.0 \%)$ & $167(18.8 \%)$ \\
$4-6$ & $92(26.0 \%)$ & $139(26.0 \%)$ & $231(26.0 \%)$ \\
$\geq 7$ & $193(54.5 \%)$ & $133(24.9 \%)$ & $326(36.7 \%)$ \\
\hline
\end{tabular}

*the values are means $\pm \mathrm{SD},{ }^{\dagger}$ number (percent), ${ }^{\ddagger}$ mean education years 
functions based on interviews with them and their family members. They did not have any of the 28 diseases that might involve cognitive decline such as stroke, seizures, and Parkinson's disease, as listed by Christensen et al. ${ }^{13}$ They also had scores that were higher than the mean minus 1SD of the Korean version of the MMSE (K-MMSE) in the respective ageand education-matched population..$^{14}$ In addition, all of the subjects had an average score of 0.42 or less on the Korean Instrumental Activities of Daily Living (IADL) instrument, which is known to discriminate dementia from normal aging. ${ }^{15}$

The patients were either enrolled from senior welfare centers in three rural areas and four cities, or were the spouses of the dementia patients. The study protocol and informed consent form were approved by the Institutional Review Board. The literate and illiterate subjects provided written and verbal informed consent to participate, respectively, prior to the start of the study.

\section{The LICA procedure}

The LICA was developed by 3 psychiatrists, 4 neurologists, and 1 neuropsychologist, and consists of 13 subtests, with possible total scores ranging from 0 to 300 . The development process, construct, sensitivity, and reliability of this tool have been reported elsewhere. ${ }^{11}$ The LICA, K-MMSE, and Clinical Dementia Rating scale were administered at each site by trained mental-health personnel on the same day.

\section{Statistical analysis}

The relative contribution of age, education/literacy, and sex to each of the LICA subtest scores was assessed using separate bivariate linear regression analyses. Age, education/literacy, and sex were coded as categorical variables. Age was categorized into five groups of ' $60-64,65-69,70-74,75-80$, and $\geq 80$ years'; education/literacy was categorized into four groups of 'illiteracy, 0-3, 4-6, and $\geq 7$ years'; and sex into two groups of 'male and female, respectively. A series of $5 \times 4 \times 2$ analyses of variance (ANOVA) were also performed to determine any main effects and interactions of age (60-64, 65-69, $70-74,75-80$, and $\geq 80$ years), education/literacy (illiteracy, $0-3,4-6$, and $\geq 7$ years), and sex in the test. Normative data including mean, standard deviation, median, and range of the 25-75th percentiles estimated from age and education/literacy were calculated. All of the analyses were performed using SPSS for Windows. The cutoff for statistical significance was set at $\mathrm{p}<0.05$.

\section{RESULTS}

\section{Demographic characteristics of the subjects}

The demographic characteristics of the 888 subjects who
Table 2. Bivariate linear regression of age, education/literacy and sex on the cognitive tests in LICA

\begin{tabular}{|c|c|c|c|}
\hline Test & Age & $\begin{array}{c}\text { Education/ } \\
\text { literacy }\end{array}$ & Sex \\
\hline \multicolumn{4}{|c|}{ Story immediate recall } \\
\hline $\mathrm{B}$ & -0.521 & 0.671 & 0.662 \\
\hline SE (B) & 0.103 & 0.112 & 0.262 \\
\hline$\beta$ & -0.167 & 0.197 & 0.085 \\
\hline $\mathrm{R}^{2}$ & 0.028 & 0.039 & 0.007 \\
\hline $\mathrm{p}$ value & $<0.001$ & $<0.001$ & 0.011 \\
\hline \multicolumn{4}{|c|}{ Word immediate recall } \\
\hline $\mathrm{B}$ & -1.035 & 0.779 & 1.218 \\
\hline SE (B) & 0.098 & 0.111 & 0.258 \\
\hline$\beta$ & -0.334 & 0.230 & 0.157 \\
\hline $\mathrm{R}^{2}$ & 0.112 & 0.053 & 0.025 \\
\hline $\mathrm{p}$ value & $<0.001$ & $<0.001$ & $<0.001$ \\
\hline \multicolumn{4}{|c|}{ Forward visuospatial span } \\
\hline B & -0.097 & 0.191 & -0.149 \\
\hline SE (B) & 0.024 & 0.026 & 0.061 \\
\hline$\beta$ & -0.134 & 0.240 & -0.082 \\
\hline $\mathrm{R}^{2}$ & 0.018 & 0.057 & 0.007 \\
\hline $\mathrm{p}$ value & $<0.001$ & $<0.001$ & 0.015 \\
\hline \multicolumn{4}{|c|}{ Backward visuospatial span } \\
\hline $\mathrm{B}$ & -0.203 & 0.439 & -0.612 \\
\hline SE (B) & 0.031 & 0.032 & 0.077 \\
\hline$\beta$ & -0.215 & 0.424 & -0.258 \\
\hline $\mathrm{R}^{2}$ & 0.046 & 0.180 & 0.067 \\
\hline$p$ value & $<0.001$ & $<0.001$ & $<0.001$ \\
\hline \multicolumn{4}{|l|}{ Digit stroop test } \\
\hline B & -0.495 & 0.678 & -0.252 \\
\hline SE (B) & 0.093 & 0.101 & 0.235 \\
\hline$\beta$ & -0.178 & 0.222 & -0.036 \\
\hline $\mathrm{R}^{2}$ & 0.032 & 0.049 & 0.001 \\
\hline $\mathrm{p}$ value & $<0.001$ & $<0.001$ & 0.284 \\
\hline \multicolumn{4}{|l|}{ Calculation } \\
\hline B & -0.591 & 1.759 & -1.852 \\
\hline SE (B) & 0.114 & 0.112 & 0.284 \\
\hline$\beta$ & -0.172 & 0.467 & -0.214 \\
\hline $\mathrm{R}^{2}$ & 0.030 & 0.218 & 0.046 \\
\hline $\mathrm{p}$ value & $<0.001$ & $<0.001$ & $<0.001$ \\
\hline \multicolumn{4}{|c|}{ Story delayed recall } \\
\hline B & -0.553 & 0.661 & 0.406 \\
\hline SE (B) & 0.104 & 0.114 & 0.266 \\
\hline$\beta$ & -0.175 & 0.191 & 0.051 \\
\hline $\mathrm{R}^{2}$ & 0.031 & 0.037 & 0.003 \\
\hline$p$ value & $<0.001$ & $<0.001$ & 0.127 \\
\hline
\end{tabular}


Table 2. Bivariate linear regression of age, education/literacy and sex on the cognitive tests in LICA (continued)

\begin{tabular}{|c|c|c|c|}
\hline Test & Age & $\begin{array}{c}\text { Education/ } \\
\text { literacy }\end{array}$ & Sex \\
\hline \multicolumn{4}{|l|}{ Story recognition } \\
\hline $\mathrm{B}$ & -0.372 & 0.490 & 0.000 \\
\hline SE (B) & 0.057 & 0.062 & 0.147 \\
\hline$\beta$ & -0.214 & 0.257 & 0.000 \\
\hline $\mathrm{R}^{2}$ & 0.046 & 0.066 & 0.000 \\
\hline $\mathrm{p}$ value & $<0.001$ & $<0.001$ & 0.999 \\
\hline \multicolumn{4}{|c|}{ Visual construction } \\
\hline B & -0.285 & 0.599 & -0.619 \\
\hline SE (B) & 0.040 & 0.040 & 0.100 \\
\hline$\beta$ & -0.234 & 0.449 & -0.203 \\
\hline $\mathrm{R}^{2}$ & 0.055 & 0.202 & 0.041 \\
\hline $\mathrm{p}$ value & $<0.001$ & $<0.001$ & $<0.001$ \\
\hline \multicolumn{4}{|c|}{ Visual recognition } \\
\hline B & -0.420 & 0.503 & -0.255 \\
\hline SE (B) & 0.054 & 0.058 & 0.139 \\
\hline$\beta$ & -0.254 & 0.278 & -0.062 \\
\hline $\mathrm{R}^{2}$ & 0.065 & 0.077 & 0.004 \\
\hline $\mathrm{p}$ value & $<0.001$ & $<0.001$ & 0.067 \\
\hline \multicolumn{4}{|c|}{ Word delayed recall } \\
\hline B & -0.520 & 0.172 & 0.836 \\
\hline SE (B) & 0.057 & 0.065 & 0.148 \\
\hline$\beta$ & -0.291 & 0.088 & 0.187 \\
\hline $\mathrm{R}^{2}$ & 0.085 & 0.008 & 0.035 \\
\hline $\mathrm{p}$ value & $<0.001$ & 0.009 & $<0.001$ \\
\hline \multicolumn{4}{|c|}{ Word recognition test } \\
\hline $\mathrm{B}$ & -0.385 & 0.217 & 0.312 \\
\hline SE (B) & 0.058 & 0.064 & 0.148 \\
\hline$\beta$ & -0.219 & 0.113 & 0.071 \\
\hline $\mathrm{R}^{2}$ & 0.048 & 0.013 & 0.005 \\
\hline $\mathrm{p}$ value & $<0.001$ & 0.001 & 0.036 \\
\hline \multicolumn{4}{|l|}{ Animal fluency } \\
\hline B & -0.823 & 1.269 & -1.567 \\
\hline SE (B) & 0.121 & 0.129 & 0.307 \\
\hline$\beta$ & -0.224 & 0.314 & -0.169 \\
\hline $\mathrm{R}^{2}$ & 0.050 & 0.099 & 0.029 \\
\hline $\mathrm{p}$ value & $<0.001$ & $<0.001$ & $<0.001$ \\
\hline \multicolumn{4}{|l|}{$\begin{array}{l}\text { Color and object } \\
\text { recognition test }\end{array}$} \\
\hline B & -0.427 & 0.385 & 0.143 \\
\hline SE (B) & 0.048 & 0.053 & 0.125 \\
\hline$\beta$ & -0.288 & 0.237 & 0.039 \\
\hline $\mathrm{R}^{2}$ & 0.083 & 0.056 & 0.001 \\
\hline $\mathrm{p}$ value & $<0.001$ & $<0.001$ & 0.252 \\
\hline
\end{tabular}

Table 2. Bivariate linear regression of age, education/literacy and sex on the cognitive tests in LICA (continued)

\begin{tabular}{|c|c|c|c|}
\hline Test & Age & $\begin{array}{c}\text { Education/ } \\
\text { literacy }\end{array}$ & Sex \\
\hline \multicolumn{4}{|l|}{ Naming } \\
\hline B & -0.409 & 0.285 & 0.260 \\
\hline SE (B) & 0.042 & 0.047 & 0.110 \\
\hline$\beta$ & -0.313 & 0.199 & 0.079 \\
\hline $\mathrm{R}^{2}$ & 0.098 & 0.040 & 0.006 \\
\hline $\mathrm{p}$ value & $<0.001$ & $<0.001$ & 0.018 \\
\hline \multicolumn{4}{|l|}{ LICA total score } \\
\hline B & -8.091 & 9.586 & -0.811 \\
\hline SE (B) & 0.630 & 0.681 & 1.716 \\
\hline$\beta$ & -0.399 & 0.431 & -0.016 \\
\hline $\mathrm{R}^{2}$ & 0.160 & 0.186 & 0.000 \\
\hline $\mathrm{p}$ value & $<0.001$ & $<0.001$ & 0.636 \\
\hline \multicolumn{4}{|l|}{ MMSE } \\
\hline B & -0.766 & 1.954 & -1.917 \\
\hline SE (B) & 0.088 & 0.075 & 0.220 \\
\hline$\beta$ & -0.282 & 0.657 & -0.281 \\
\hline $\mathrm{R}^{2}$ & 0.080 & 0.432 & 0.079 \\
\hline $\mathrm{p}$ value & $<0.001$ & $<0.001$ & $<0.001$ \\
\hline
\end{tabular}

Independent variables; age, education/literacy and sex. Age is categorized as ' $60-64,65-69,70-74,75-80$, and $\geq 80$ years'; education/literacy is categorized as 'illiteracy, $0-3,4-6$, and $\geq 7$ years'; and sex as 'male and female', respectively. LICA: literacy independent cognitive assessment, MMSE: mini mental state examination, B: regression coefficient, SE (B): standard error of B, $\beta$ : standardized regression coefficient, $R^{2}$ : variance explained by each variable

completed the LICA are given in Table 1. The mean age of the male subjects (72.8 years) did not differ significantly from that of the female subjects ( 72.1 years; $t=1.45, \mathrm{p}=0.148$ ), but the mean number of years of education was much higher in the male group ( 8.4 years) than in the female group ( 4.5 years; $\mathrm{t}=11.8, \mathrm{p}<0.001)$. The proportion of illiterate subjects differed significantly between the males (7.3\%) and females (27.0\%; $\chi^{2}=52.9, \mathrm{p}<0.001$ ).

\section{Effects of age, education/literacy, and sex on cognitive tests in the LICA}

Bivariate linear regression analyses revealed that all 15 of the cognitive tests (13 subtests plus 2 subcategories of subtests) in the LICA, the total LICA score, and the total MMSE score were significantly influenced by both age and education/literacy, as indicated in Table 2 . In every test, the younger and more-educated subjects outperformed the older and illiterate/less-educated subjects, respectively.

The gender effect differed for each test (Table 2). Males performed significantly better than females in forward visuospa- 
Table 3. Analyses of variance for main effects and interactions of age, education/literacy, and sex on the cognitive tests in LICA

\begin{tabular}{|c|c|c|c|c|}
\hline \multirow{2}{*}{ Test } & \multicolumn{2}{|c|}{ Main effect } & \multicolumn{2}{|c|}{ Interaction } \\
\hline & Variable & $\mathrm{F}, \mathrm{p}$ value* & Variable & $\mathrm{F}, \mathrm{p}$ value $^{\dagger}$ \\
\hline \multirow[t]{3}{*}{ Story immediate recall } & Age & $\mathrm{F}=7.64, \mathrm{p}<0.001$ & Age $\times$ Edu/Lit & $\mathrm{F}=0.67, \mathrm{p}=0.781$ \\
\hline & Edu/Lit & $\mathrm{F}=15.19, \mathrm{p}<0.001$ & Age $\times$ Sex & $\mathrm{F}=2.52, \mathrm{p}=0.040$ \\
\hline & Sex & $\mathrm{F}=6.42, \mathrm{p}=0.011$ & Edu/Lit $\times$ Sex & $\mathrm{F}=1.00, \mathrm{p}=0.392$ \\
\hline \multirow[t]{3}{*}{ Word immediate recall } & Age & $\mathrm{F}=28.46, \mathrm{p}<0.001$ & Age $\times$ Edu/Lit & $\mathrm{F}=0.98, \mathrm{p}=0.464$ \\
\hline & Edu/Lit & $\mathrm{F}=19.20, \mathrm{p}<0.001$ & Age $\times$ Sex & $\mathrm{F}=0.15, \mathrm{p}=0.965$ \\
\hline & Sex & $\mathrm{F}=22.36, \mathrm{p}<0.001$ & Edu/Lit $\times$ Sex & $\mathrm{F}=4.19, \mathrm{p}=0.006$ \\
\hline \multirow[t]{3}{*}{ Forward visuospatial span } & Age & $\mathrm{F}=4.95, \mathrm{p}=0.001$ & Age $\times$ Edu/Lit & $\mathrm{F}=0.77, \mathrm{p}=0.681$ \\
\hline & Edu/Lit & $\mathrm{F}=18.78, \mathrm{p}<0.001$ & Age $\times$ Sex & $\mathrm{F}=0.63, \mathrm{p}=0.640$ \\
\hline & Sex & $\mathrm{F}=5.98, \mathrm{p}=0.015$ & Edu/Lit $\times$ Sex & $\mathrm{F}=1.29, \mathrm{p}=0.276$ \\
\hline \multirow[t]{3}{*}{ Backward visuospatial span } & Age & $\mathrm{F}=12.07, \mathrm{p}<0.001$ & Age $\times$ Edu/Lit & $\mathrm{F}=0.71, \mathrm{p}=0.739$ \\
\hline & Edu/Lit & $\mathrm{F}=65.73, \mathrm{p}<0.001$ & Age $\times$ Sex & $\mathrm{F}=0.46, \mathrm{p}=0.768$ \\
\hline & Sex & $\mathrm{F}=63.10, \mathrm{p}<0.001$ & Edu/Lit $\times$ Sex & $\mathrm{F}=0.72, \mathrm{p}=0.541$ \\
\hline \multirow[t]{3}{*}{ Digit stroop test } & Age & $\mathrm{F}=9.81, \mathrm{p}<0.001$ & Age $\times$ Edu/Lit & $\mathrm{F}=1.56, \mathrm{p}=0.099$ \\
\hline & Edu/Lit & $\mathrm{F}=18.08, \mathrm{p}<0.001$ & Age $\times$ Sex & $\mathrm{F}=1.00, \mathrm{p}=0.405$ \\
\hline & Sex & $\mathrm{F}=1.15, \mathrm{p}=0.284$ & Edu/Lit $\times$ Sex & $\mathrm{F}=2.29, \mathrm{p}=0.077$ \\
\hline \multirow[t]{3}{*}{ Calculation } & Age & $\mathrm{F}=7.98, \mathrm{p}<0.001$ & Age $\times$ Edu/Lit & $\mathrm{F}=2.30, \mathrm{p}=0.007$ \\
\hline & Edu/Lit & $\mathrm{F}=103.79, \mathrm{p}<0.001$ & Age $\times$ Sex & $\mathrm{F}=0.58, \mathrm{p}=0.680$ \\
\hline & Sex & $\mathrm{F}=42.60, \mathrm{p}<0.001$ & Edu/Lit $\times$ Sex & $\mathrm{F}=1.09, \mathrm{p}=0.354$ \\
\hline \multirow[t]{3}{*}{ Story delayed recall } & Age & $\mathrm{F}=7.74, \mathrm{p}<0.001$ & Age $\times$ Edu/Lit & $\mathrm{F}=0.55, \mathrm{p}=0.882$ \\
\hline & Edu/Lit & $\mathrm{F}=15.99, \mathrm{p}<0.001$ & Age $\times$ Sex & $\mathrm{F}=2.35, \mathrm{p}=0.053$ \\
\hline & Sex & $\mathrm{F}=2.33, \mathrm{p}=0.127$ & $\mathrm{Edu} / \mathrm{Lit} \times \operatorname{Sex}$ & $\mathrm{F}=0.84, \mathrm{p}=0.470$ \\
\hline \multirow[t]{3}{*}{ Story recognition } & Age & $\mathrm{F}=11.23, \mathrm{p}<0.001$ & Age $\times$ Edu/Lit & $\mathrm{F}=1.29, \mathrm{p}=0.218$ \\
\hline & Edu/Lit & $\mathrm{F}=26.21, \mathrm{p}<0.001$ & Age $\times$ Sex & $\mathrm{F}=2.33, \mathrm{p}=0.054$ \\
\hline & Sex & $\mathrm{F}=0.00, \mathrm{p}=0.999$ & Edu/Lit $\times$ Sex & $\mathrm{F}=0.65, \mathrm{p}=0.583$ \\
\hline \multirow[t]{3}{*}{ Visual construction } & Age & $\mathrm{F}=14.13, \mathrm{p}<0.001$ & Age $\times$ Edu/Lit & $\mathrm{F}=0.91, \mathrm{p}=0.534$ \\
\hline & Edu/Lit & $\mathrm{F}=86.72, \mathrm{p}<0.001$ & Age $\times$ Sex & $\mathrm{F}=2.46, \mathrm{p}=0.044$ \\
\hline & Sex & $\mathrm{F}=37.93, \mathrm{p}<0.001$ & Edu/Lit $\times$ Sex & $\mathrm{F}=3.93, \mathrm{p}=0.008$ \\
\hline \multirow[t]{3}{*}{ Visual recognition } & Age & $\mathrm{F}=10.2, \mathrm{p}<0.001$ & Age $\times$ Edu/Lit & $\mathrm{F}=0.62, \mathrm{p}=0.829$ \\
\hline & Edu/Lit & $\mathrm{F}=15.6, \mathrm{p}<0.001$ & Age $\times$ Sex & $\mathrm{F}=0.23, \mathrm{p}=0.923$ \\
\hline & Sex & $\mathrm{F}=0.43, \mathrm{p}<0.513$ & Edu/Lit $\times$ Sex & $\mathrm{F}=0.49, \mathrm{p}=0.689$ \\
\hline \multirow[t]{3}{*}{ Word delayed recall } & Age & $\mathrm{F}=20.63, \mathrm{p}<0.001$ & Age $\times$ Edu/Lit & $\mathrm{F}=0.75, \mathrm{p}=0.707$ \\
\hline & Edu/Lit & $\mathrm{F}=4.48, \mathrm{p}=0.004$ & Age $\times$ Sex & $\mathrm{F}=0.74, \mathrm{p}=0.568$ \\
\hline & Sex & $\mathrm{F}=32.01, \mathrm{p}<0.001$ & Edu/Lit $\times$ Sex & $\mathrm{F}=0.68, \mathrm{p}=0.566$ \\
\hline \multirow[t]{3}{*}{ Word recognition test } & Age & $\mathrm{F}=11.23, \mathrm{p}<0.001$ & Age $\times$ Edu/Lit & $\mathrm{F}=0.51, \mathrm{p}=0.908$ \\
\hline & Edu/Lit & $\mathrm{F}=6.13, \mathrm{p}<0.001$ & Age $\times$ Sex & $\mathrm{F}=0.12, \mathrm{p}=0.976$ \\
\hline & Sex & $\mathrm{F}=4.43, \mathrm{p}=0.036$ & $\mathrm{Edu} / \mathrm{Lit} \times \operatorname{Sex}$ & $\mathrm{F}=1.14, \mathrm{p}=0.334$ \\
\hline \multirow[t]{3}{*}{ Animal fluency } & Age & $\mathrm{F}=12.69, \mathrm{p}<0.001$ & Age $\times$ Edu/Lit & $\mathrm{F}=1.17, \mathrm{p}=0.303$ \\
\hline & Edu/Lit & $\mathrm{F}=37.81, \mathrm{p}<0.001$ & Age $\times$ Sex & $\mathrm{F}=0.30, \mathrm{p}=0.881$ \\
\hline & Sex & $\mathrm{F}=26.06, \mathrm{p}<0.001$ & Edu/Lit $\times$ Sex & $\mathrm{F}=0.08, \mathrm{p}=0.972$ \\
\hline \multirow[t]{3}{*}{ Color and object recognition test } & Age & $\mathrm{F}=60.61, \mathrm{p}<0.001$ & Age $\times$ Edu/Lit & $\mathrm{F}=1.07, \mathrm{p}=0.385$ \\
\hline & Edu/Lit & $\mathrm{F}=22.06, \mathrm{p}<0.001$ & Age $\times$ Sex & $\mathrm{F}=2.51, \mathrm{p}=0.040$ \\
\hline & Sex & $\mathrm{F}=1.31, \mathrm{p}=0.252$ & Edu/Lit $\times$ Sex & $\mathrm{F}=0.40, \mathrm{p}=0.756$ \\
\hline
\end{tabular}


Table 3. Analyses of variance for main effects and interactions of age, education/literacy, and sex on the cognitive tests in LICA (continued)

\begin{tabular}{|c|c|c|c|c|}
\hline \multirow{2}{*}{ Test } & \multicolumn{2}{|c|}{ Main effect } & \multicolumn{2}{|c|}{ Interaction } \\
\hline & Variable & F, p value* & Variable & F, p value ${ }^{\dagger}$ \\
\hline \multirow[t]{3}{*}{ Naming } & Age & $\mathrm{F}=24.82, \mathrm{p}<0.001$ & Age $\times$ Edu/Lit & $\mathrm{F}=3.24, \mathrm{p}<0.001$ \\
\hline & Edu/Lit & $\mathrm{F}=18.43, \mathrm{p}<0.001$ & Age $\times$ Sex & $\mathrm{F}=3.12, \mathrm{p}=0.015$ \\
\hline & Sex & $\mathrm{F}=5.58, \mathrm{p}=0.018$ & Edu/Lit $\times$ Sex & $\mathrm{F}=0.94, \mathrm{p}=0.423$ \\
\hline \multirow[t]{3}{*}{ LICA total score } & Age & $\mathrm{F}=41.97, \mathrm{p}<0.001$ & Age $\times$ Edu/Lit & $\mathrm{F}=0.71, \mathrm{p}=0.743$ \\
\hline & Edu/Lit & $\mathrm{F}=75.15, \mathrm{p}<0.001$ & Age $\times$ Sex & $\mathrm{F}=0.39, \mathrm{p}=0.817$ \\
\hline & Sex & $\mathrm{F}=0.22, \mathrm{p}=0.636$ & Edu/Lit $\times$ Sex & $\mathrm{F}=1.26, \mathrm{p}=0.287$ \\
\hline \multirow[t]{3}{*}{ MMSE } & Age & $\mathrm{F}=20.71, \mathrm{p}<0.001$ & Age $\times$ Edu/Lit & $\mathrm{F}=0.97, \mathrm{p}=0.476$ \\
\hline & Edu/Lit & $\mathrm{F}=245.92, \mathrm{p}<0.001$ & Age $\times$ Sex & $\mathrm{F}=0.80, \mathrm{p}=0.526$ \\
\hline & Sex & $\mathrm{F}=76.03, \mathrm{p}<0.001$ & Edu/Lit $\times$ Sex & $\mathrm{F}=5.10, \mathrm{p}=0.002$ \\
\hline
\end{tabular}

Independent variables; age, education/literacy and sex. Age is categorized as ‘60-64, 65-69, 70-74, 75-80, and $\geq 80$ years'; education/literacy as 'illiteracy, $0-3,4-6$, and $\geq 7$ years'; and sex as 'male and female,' respectively. *analyzed by analyses of variance (ANOVA), tanalyzed by three-way ANOVA. LICA: literacy independent cognitive assessment, Edu: education, Lit: literacy, MMSE: mini mental state examination

tial span, backward visuospatial span, calculation, visual construction, animal fluency, and the MMSE. In contrast, females significantly outperformed males in story immediate recall, word immediate recall, word delayed recall, word recognition test, and naming. There were no significant differences between the genders for the digit Stroop test, story delayed recall, story recognition, visual recognition, Color and Object Recognition Test, and LICA total score.

As shown in Table 3, ANOVA tests revealed a significant interaction between age and gender for the story immediate recall, visual construction, CORT, and naming. The test performance for these story immediate recall, CORT, and naming declined more rapidly in men than in women with advancing age, however, test performance for visual construction declined more prominently in women than in men with advancing age. The education and gender had a significant interaction for word immediate recall, visual construction, and MMSE. ANOVA tests also showed a significant interaction between education/literacy and gender for the word immediate recall, visual construction, and MMSE. The test performance for these three tests increased more rapidly in women than in men with increasing education.

\section{Normative data}

The findings for the effects of demographic variables on test performance indicated that age and education should be taken into account when attempting to accurately interpret the LICA cognitive subtests. Therefore, the total group was divided into five age groups (60-64, 65-69, 70-74, 75-80, and $\geq 80$ years) and four educational levels (illiterate, and 0-3, 4-6, and $\geq 7$ years of education). The normative scores for each stratified cell are presented in the form of a mean and standard deviation, and a median and range from the 25 th to the 75 th per- centile (Table 4).

\section{DISCUSSION}

To the best of our knowledge, the LICA is the first neuropsychological test that has been developed specifically for the illiterate elderly population. Although each subtest of neuropsychological batteries have been standardized for the illiterate population in several countries, a mass standardization study-examining both illiterate and literate population-has yet to be conducted on a neuropsychological test battery developed for illiterate people. ${ }^{16-25}$

The results of the present study suggest that older age is associated with lower performance on all cognitive tests in the LICA, indicating that the cognitive functions assessed by the LICA gradually decline with advancing age even within the elderly. This decline in cognitive function has also been shown for many other neuropsychological tests. The normative studies on the Korean version of the Consortium to Establish a Registry for Alzheimer's Disease Assessment Packet (CERAD-K) conducted in Korea found a similar impact of age on most cognitive tests. ${ }^{26}$

With regard to education, the present results suggest that a higher educational level is associated with a better performance in all of the LICA subtests. The duration of education affect the performance in most neuropsychological tests, as verified in a normative study of CERAD-K conducted in Korea [i.e. the duration of education significantly impacted the performance score in all of the categories (verbal fluency, Boston naming test, MMSE-KC, word list memory, constructional praxis, word list recall, constructional recall) except word list recognition]. ${ }^{26}$ Although the LICA was expected to be less significantly affected by either illiteracy or education 
Table 4. LICA normative data according to age, literacy and education level

\begin{tabular}{|c|c|c|c|c|c|}
\hline \multirow{2}{*}{ Test } & \multirow{2}{*}{ Age } & \multirow{2}{*}{ Illiteracy } & \multicolumn{3}{|c|}{ Education (years) } \\
\hline & & & $0-3$ & $4-6$ & $\geq 7$ \\
\hline \multirow[t]{10}{*}{ Story immediate recall } & $60-64$ & $5.9 \pm 2.6^{*}$ & $9.6 \pm 3.2$ & $8.4 \pm 3.2$ & $9.6 \pm 4.3$ \\
\hline & & $6.0(4.0-8.0)^{\dagger}$ & $9.8(7.0-11.0)$ & $9.0(5.0-11.0)$ & $9.0(7.0-12.5)$ \\
\hline & $65-69$ & $7.2 \pm 3.6$ & $8.0 \pm 3.6$ & $8.1 \pm 4.0$ & $9.5 \pm 4.2$ \\
\hline & & $7.5(4.0-9.0)$ & $7.5(5.0-11.0)$ & $7.0(5.0-12.0)$ & $9.0(6.0-13.0)$ \\
\hline & $70-74$ & $6.5 \pm 2.7$ & $7.4 \pm 3.0$ & $7.0 \pm 3.6$ & $8.2 \pm 4.1$ \\
\hline & & $7.0(5.0-8.0)$ & $6.0(5.0-9.3)$ & $7.0(4.0-9.0)$ & $8.0(5.0-11.0)$ \\
\hline & $75-79$ & $7.2 \pm 3.3$ & $7.9 \pm 3.3$ & $6.6 \pm 3.7$ & $8.7 \pm 4.3$ \\
\hline & & $6.0(5.0-9.0)$ & $8.0(5.0-10.0)$ & $6.0(4.0-9.3)$ & $9.0(6.0-11.0)$ \\
\hline & $\geq 80$ & $6.0 \pm 3.0$ & $6.3 \pm 2.5$ & $6.9 \pm 4.0$ & $7.9 \pm 4.6$ \\
\hline & & $6.0(4.0-7.0)$ & $6.0(4.5-8.0)$ & $6.0(4.0-8.0)$ & $6.3(4.8-10.9)$ \\
\hline \multirow[t]{10}{*}{ Word immediate recall } & $60-64$ & $14.7 \pm 4.0$ & $18.4 \pm 4.5$ & $18.0 \pm 3.1$ & $18.6 \pm 4.0$ \\
\hline & & $14.0(12.0-18.0)$ & $18.5(14.3-22.8)$ & $17.0(16.0-21.0)$ & $19.0(16.0-22.0)$ \\
\hline & $65-69$ & $16.3 \pm 3.7$ & $17.2 \pm 3.0$ & $16.5 \pm 3.3$ & $18.2 \pm 3.2$ \\
\hline & & $16.0(13.5-19.0)$ & $17.0(16.0-19.5)$ & $17.0(14.0-19.0)$ & $18.0(16.0-20.5)$ \\
\hline & $70-74$ & $14.5 \pm 3.6$ & $15.6 \pm 3.7$ & $16.0 \pm 3.5$ & $16.8 \pm 3.3$ \\
\hline & & $14.0(12.0-17.0)$ & $15.5(13.0-17.3)$ & $16.0(14.0-18.0)$ & $17.0(15.0-19.0)$ \\
\hline & $75-79$ & $14.0 \pm 3.9$ & $15.9 \pm 3.6$ & $14.6 \pm 3.3$ & $15.7 \pm 3.4$ \\
\hline & & $14.0(11.0-17.0)$ & $16.0(13.0-18.0)$ & $14.0(12.0-17.0)$ & $16.0(14.0-18.0)$ \\
\hline & $\geq 80$ & $13.4 \pm 3.1$ & $13.9 \pm 4.2$ & $14.7 \pm 3.3$ & $14.4 \pm 4.2$ \\
\hline & & $14.0(11.0-16.0)$ & $14.0(11.0-17.0)$ & $15.0(12.0-18.0)$ & $13.0(11.8-16.3)$ \\
\hline \multirow[t]{10}{*}{ Forward visuospatial span } & $60-64$ & $4.7 \pm 0.6$ & $4.9 \pm 0.9$ & $5.0 \pm 0.6$ & $5.0 \pm 0.8$ \\
\hline & & $5.0(4.0-5.0)$ & $5.0(4.0-5.8)$ & $5.0(5.0-5.0)$ & $5.0(4.0-5.5)$ \\
\hline & $65-69$ & $4.6 \pm 0.8$ & $4.8 \pm 0.9$ & $5.0 \pm 0.8$ & $5.3 \pm 1.0$ \\
\hline & & $4.0(4.0-5.0)$ & $5.0(4.0-5.0)$ & $5.0(4.0-5.0)$ & $5.0(5.0-6.0)$ \\
\hline & $70-74$ & $4.5 \pm 0.8$ & $4.8 \pm 0.7$ & $5.0 \pm 1.0$ & $5.1 \pm 0.8$ \\
\hline & & $4.0(4.0-5.0)$ & $5.0(4.0-5.0)$ & $5.0(4.0-5.0)$ & $5.0(4.0-6.0)$ \\
\hline & $75-79$ & $4.6 \pm 0.9$ & $4.6 \pm 0.8$ & $4.6 \pm 0.8$ & $5.0 \pm 1.0$ \\
\hline & & $4.0(4.0-5.0)$ & $5.0(4.0-5.0)$ & $5.0(4.0-5.0)$ & $5.0(4.0-6.0)$ \\
\hline & $\geq 80$ & $4.4 \pm 0.7$ & $4.8 \pm 0.8$ & $4.7 \pm 0.9$ & $5.0 \pm 0.9$ \\
\hline & & $4.0(4.0-5.0)$ & $5.0(4.0-5.0)$ & $5.0(4.0-5.0)$ & $5.0(4.0-5.5)$ \\
\hline \multirow[t]{10}{*}{ Backward visuospatial span } & $60-64$ & $3.5 \pm 0.7$ & $3.5 \pm 0.8$ & $4.4 \pm 0.9$ & $4.6 \pm 1.2$ \\
\hline & & $4.0(3.0-4.0)$ & $3.0(3.0-4.0)$ & $4.0(4.0-5.0)$ & $4.0(4.0-5.0)$ \\
\hline & $65-69$ & $3.4 \pm 0.9$ & $3.7 \pm 1.0$ & $4.5 \pm 1.1$ & $4.4 \pm 1.1$ \\
\hline & & $3.0(3.0-4.0)$ & $4.0(3.0-4.0)$ & $4.0(4.0-5.0)$ & $4.0(4.0-5.0)$ \\
\hline & $70-74$ & $3.1 \pm 0.9$ & $3.8 \pm 1.0$ & $4.2 \pm 0.9$ & $4.6 \pm 1.2$ \\
\hline & & $3.0(3.0-4.0)$ & $4.0(3.0-4.0)$ & $4.0(4.0-5.0)$ & $4.0(4.0-6.0)$ \\
\hline & $75-79$ & $3.1 \pm 1.1$ & $3.4 \pm 1.0$ & $3.9 \pm 1.0$ & $4.3 \pm 1.0$ \\
\hline & & $3.0(3.0-4.0)$ & $3.0(3.0-4.0)$ & $4.0(3.0-5.0)$ & $4.0(4.0-5.0)$ \\
\hline & $\geq 80$ & $2.8 \pm 0.7$ & $3.4 \pm 0.9$ & $3.7 \pm 1.0$ & $4.1 \pm 1.1$ \\
\hline & & $3.0(3.0-3.0)$ & $3.0(3.0-4.0)$ & $4.0(3.0-4.0)$ & $4.0(3.0-5.0)$ \\
\hline \multirow[t]{6}{*}{ Digit stroop test } & $60-64$ & $19.2 \pm 8.0$ & $22.5 \pm 2.2$ & $21.8 \pm 3.8$ & $23.2 \pm 1.5$ \\
\hline & & $23.0(15.0-24.0)$ & $23.5(21.3-24.0)$ & $23.0(22.0-24.0)$ & $23.0(22.0-24.0)$ \\
\hline & $65-69$ & $22.0 \pm 2.5$ & $22.5 \pm 2.9$ & $22.6 \pm 2.4$ & $22.6 \pm 1.8$ \\
\hline & & $23.0(21.0-24.0)$ & $23.0(22.0-24.0)$ & $23.0(22.0-24.0)$ & $23.0(22.0-24.0)$ \\
\hline & $70-74$ & $20.7 \pm 4.0$ & $22.2 \pm 3.5$ & $22.8 \pm 1.8$ & $22.6 \pm 1.9$ \\
\hline & & $22.0(18.0-24.0)$ & $23.0(22.0-24.0)$ & $23.0(22.0-24.0)$ & $23.0(22.0-24.0)$ \\
\hline
\end{tabular}


Table 4. LICA normative data according to age, literacy and education level (continued)

\begin{tabular}{|c|c|c|c|c|c|}
\hline \multirow{2}{*}{ Test } & \multirow{2}{*}{ Age } & \multirow{2}{*}{ Illiteracy } & \multicolumn{3}{|c|}{ Education (years) } \\
\hline & & & $0-3$ & $4-6$ & $\geq 7$ \\
\hline & $75-79$ & $20.4 \pm 5.1$ & $22.3 \pm 3.0$ & $21.4 \pm 3.5$ & $22.6 \pm 2.3$ \\
\hline & & $22.0(18.5-24.0)$ & $23.0(21.0-24.0)$ & $23.0(20.5-23.0)$ & $23.0(22.0-24.0)$ \\
\hline & $\geq 80$ & $18.6 \pm 6.4$ & $20.1 \pm 4.6$ & $20.7 \pm 4.5$ & $22.2 \pm 2.4$ \\
\hline & & $21.0(17.0-24.0)$ & $22.0(18.0-23.0)$ & $22.0(20.0-23.0)$ & $23.0(21.0-24.0)$ \\
\hline \multirow[t]{10}{*}{ Calculation } & $60-64$ & $18.6 \pm 6.4$ & $21.2 \pm 3.2$ & $22.0 \pm 2.7$ & $23.7 \pm 0.9$ \\
\hline & & $20.0(17.0-23.0)$ & $22.0(19.3-24.0)$ & $24.0(19.0-24.0)$ & $24.0(24.0-24.0)$ \\
\hline & $65-69$ & $19.2 \pm 5.5$ & $22.1 \pm 2.9$ & $23.0 \pm 1.7$ & $23.3 \pm 1.8$ \\
\hline & & $21.0(16.0-24.0)$ & $24.0(20.5-24.0)$ & $24.0(22.8-24.0)$ & $24.0(24.0-24.0)$ \\
\hline & $70-74$ & $16.4 \pm 7.1$ & $21.8 \pm 2.4$ & $22.7 \pm 2.0$ & $23.2 \pm 1.4$ \\
\hline & & $18.0(11.0-23.0)$ & $23.0(20.0-24.0)$ & $24.0(22.0-24.0)$ & $24.0(23.0-24.0)$ \\
\hline & $75-79$ & $18.7 \pm 6.8$ & $21.0 \pm 4.5$ & $22.2 \pm 3.1$ & $23.0 \pm 2.1$ \\
\hline & & $22.0(16.0-24.0)$ & $22.0(20.0-24.0)$ & $24.0(22.0-24.0)$ & $24.0(24.0-24.0)$ \\
\hline & $\geq 80$ & $14.8 \pm 7.0$ & $21.5 \pm 3.1$ & $21.8 \pm 2.9$ & $23.0 \pm 2.2$ \\
\hline & & $15.0(12.0-21.0)$ & $23.0(19.0-24.0)$ & $23.0(22.0-24.0)$ & $24.0(23.0-24.0)$ \\
\hline \multirow[t]{10}{*}{ Story delayed recall } & $60-64$ & $3.2 \pm 2.1$ & $6.6 \pm 3.7$ & $5.4 \pm 3.4$ & $8.1 \pm 4.4$ \\
\hline & & $4.0(1.0-4.5)$ & $5.8(4.0-8.0)$ & $5.0(3.0-8.0)$ & $8.0(6.0-10.0)$ \\
\hline & $65-69$ & $5.2 \pm 3.6$ & $6.4 \pm 3.9$ & $5.7 \pm 3.6$ & $7.4 \pm 4.3$ \\
\hline & & $5.0(3.0-7.5)$ & $6.0(3.0-10.0)$ & $5.0(3.0-8.0)$ & $7.0(4.0-11.0)$ \\
\hline & $70-74$ & $4.1 \pm 2.9$ & $5.6 \pm 3.3$ & $5.3 \pm 3.3$ & $6.2 \pm 4.6$ \\
\hline & & $4.0(2.0-6.0)$ & $5.0(3.0-9.0)$ & $5.0(3.0-8.0)$ & $5.0(2.0-9.0)$ \\
\hline & $75-79$ & $5.1 \pm 3.0$ & $5.6 \pm 3.3$ & $4.4 \pm 3.7$ & $6.3 \pm 4.1$ \\
\hline & & $4.0(3.0-8.0)$ & $6.0(4.0-8.0)$ & $4.0(1.0-7.0)$ & $6.0(3.0-9.0)$ \\
\hline & $\geq 80$ & $4.2 \pm 3.5$ & $4.2 \pm 2.1$ & $4.3 \pm 3.1$ & $5.4 \pm 4.2$ \\
\hline & & $3.8(1.8-6.0)$ & $4.0(3.0-6.0)$ & $4.0(2.0-5.5)$ & $4.5(1.8-8.3)$ \\
\hline \multirow[t]{10}{*}{ Story recognition } & $60-64$ & $6.7 \pm 1.6$ & $6.9 \pm 3.7$ & $6.9 \pm 1.7$ & $7.7 \pm 1.6$ \\
\hline & & $6.0(5.0-8.0)$ & $7.0(6.0-8.0)$ & $7.0(5.0-8.0)$ & $8.0(7.0-9.0)$ \\
\hline & $65-69$ & $6.4 \pm 2.2$ & $6.6 \pm 2.0$ & $7.0 \pm 1.9$ & $7.5 \pm 1.6$ \\
\hline & & $7.0(5.0-8.0)$ & $7.0(5.5-8.0)$ & $7.0(6.0-8.0)$ & $7.0(7.0-8.5)$ \\
\hline & $70-74$ & $5.0 \pm 2.3$ & $6.7 \pm 1.8$ & $6.4 \pm 2.5$ & $7.0 \pm 2.0$ \\
\hline & & $5.0(3.0-7.0)$ & $7.0(6.0-8.0)$ & $7.0(6.0-8.0)$ & $7.0(6.0-9.0)$ \\
\hline & $75-79$ & $6.0 \pm 2.1$ & $6.4 \pm 2.1$ & $5.4 \pm 2.5$ & $7.2 \pm 1.7$ \\
\hline & & $7.0(4.0-8.0)$ & $7.0(5.0-8.0)$ & $5.0(4.0-7.0)$ & $8.0(7.0-8.0)$ \\
\hline & $\geq 80$ & $4.6 \pm 2.6$ & $6.4 \pm 1.9$ & $5.9 \pm 1.8$ & $6.5 \pm 2.4$ \\
\hline & & $5.0(2.0-7.0)$ & $6.5(6.0-8.0)$ & $6.0(4.0-7.0)$ & $7.0(5.0-8.3)$ \\
\hline \multirow[t]{10}{*}{ Visual construction } & $60-64$ & $8.5 \pm 1.8$ & $9.4 \pm 1.0$ & $9.7 \pm 0.5$ & $9.8 \pm 0.5$ \\
\hline & & $9.0(8.0-10.0)$ & $10.0(9.0-10.0)$ & $10.0(9.5-10.0)$ & $10.0(10.0-10.0)$ \\
\hline & $65-69$ & $8.5 \pm 1.6$ & $9.4 \pm 0.9$ & $9.7 \pm 0.8$ & $9.9 \pm 0.4$ \\
\hline & & $9.0(7.8-10.0)$ & $10.0(9.0-10.0)$ & $10.0(9.9-10.0)$ & $10.0(10.0-10.0)$ \\
\hline & $70-74$ & $8.0 \pm 2.0$ & $9.3 \pm 1.3$ & $9.7 \pm 1.0$ & $9.7 \pm 0.6$ \\
\hline & & $8.5(7.0-10.0)$ & $10.0(9.0-10.0)$ & $10.0(9.6-10.0)$ & $10.0(10.0-10.0)$ \\
\hline & $75-79$ & $7.3 \pm 2.7$ & $8.8 \pm 1.8$ & $9.4 \pm 0.8$ & $9.7 \pm 0.9$ \\
\hline & & $8.0(5.0-10.0)$ & $9.5(8.0-10.0)$ & $9.5(9.0-10.0)$ & $10.0(10.0-10.0)$ \\
\hline & $\geq 80$ & $7.5 \pm 2.2$ & $8.4 \pm 1.9$ & $9.1 \pm 1.4$ & $9.7 \pm 0.7$ \\
\hline & & $8.0(6.0-9.6)$ & $9.0(7.3-10.0)$ & $10.0(8.5-10.0)$ & $10.0(10.0-10.0)$ \\
\hline \multirow[t]{2}{*}{ Visual recognition } & $60-64$ & $14.6 \pm 2.5$ & $15.9 \pm 1.6$ & $16.4 \pm 1.6$ & $16.3 \pm 1.9$ \\
\hline & & $15.0(12.0-17.0)$ & $16.0(14.3-17.0)$ & $16.0(15.0-17.0)$ & $16.0(15.0-18.0)$ \\
\hline
\end{tabular}


Table 4. LICA normative data according to age, literacy and education level (continued)

\begin{tabular}{|c|c|c|c|c|c|}
\hline \multirow{2}{*}{ Test } & \multirow{2}{*}{ Age } & \multirow{2}{*}{ Illiteracy } & \multicolumn{3}{|c|}{ Education (years) } \\
\hline & & & $0-3$ & $4-6$ & $\geq 7$ \\
\hline & $65-69$ & $15.1 \pm 1.0$ & $15.5 \pm 2.0$ & $15.8 \pm 1.7$ & $15.8 \pm 2.0$ \\
\hline & & $15.0(14.0-16.0)$ & $15.0(14.0-17.0)$ & $16.0(14.0-17.0)$ & $16.0(14.0-17.0)$ \\
\hline & $70-74$ & $14.5 \pm 1.8$ & $15.7 \pm 1.7$ & $15.7 \pm 1.7$ & $16.0 \pm 1.8$ \\
\hline & & $14.0(13.0-16.0)$ & $16.0(14.8-17.0)$ & $16.0(15.0-17.0)$ & $16.0(15.0-17.0)$ \\
\hline & $75-79$ & $14.1 \pm 1.9$ & $14.7 \pm 2.3$ & $15.1 \pm 1.7$ & $15.7 \pm 1.8$ \\
\hline & & $14.0(13.0-16.0)$ & $15.0(13.0-16.0)$ & $15.0(14.0-16.0)$ & $16.0(15.0-17.0)$ \\
\hline & $\geq 80$ & $13.2 \pm 2.8$ & $14.4 \pm 2.0$ & $14.6 \pm 2.1$ & $15.2 \pm 1.8$ \\
\hline & & $14.0(12.0-15.0)$ & $15.0(13.0-16.0)$ & $15.0(13.0-16.0)$ & $15.0(14.0-16.0)$ \\
\hline \multirow[t]{10}{*}{ Word delayed recall } & $60-64$ & $5.6 \pm 2.1$ & $5.8 \pm 1.8$ & $6.4 \pm 1.6$ & $6.0 \pm 2.0$ \\
\hline & & $5.0(4.0-8.0)$ & $6.0(4.0-7.0)$ & $6.0(5.0-8.0)$ & $6.0(5.0-8.0)$ \\
\hline & $65-69$ & $5.1 \pm 2.4$ & $5.5 \pm 2.4$ & $5.2 \pm 1.9$ & $5.6 \pm 1.9$ \\
\hline & & $5.0(4.0-6.5)$ & $5.0(4.5-7.5)$ & $5.0(4.0-7.0)$ & $6.0(4.0-7.0)$ \\
\hline & $70-74$ & $4.6 \pm 2.1$ & $4.7 \pm 2.6$ & $4.8 \pm 2.3$ & $5.0 \pm 1.8$ \\
\hline & & $4.0(4.0-6.0)$ & $5.0(3.0-6.0)$ & $5.0(3.0-7.0)$ & $5.0(4.0-6.0)$ \\
\hline & $75-79$ & $4.6 \pm 2.3$ & $4.9 \pm 2.3$ & $3.8 \pm 2.3$ & $4.3 \pm 2.4$ \\
\hline & & $5.0(3.0-6.0)$ & $4.0(3.0-7.0)$ & $4.0(2.0-6.0)$ & $5.0(2.0-6.0)$ \\
\hline & $\geq 80$ & $3.5 \pm 1.6$ & $4.5 \pm 1.7$ & $3.5 \pm 2.1$ & $4.4 \pm 2.0$ \\
\hline & & $3.5(2.0-5.0)$ & $5.0(4.0-5.0)$ & $4.0(2.0-5.0)$ & $5.0(2.8-6.0)$ \\
\hline \multirow[t]{10}{*}{ Word recognition test } & $60-64$ & $18.3 \pm 1.8$ & $19.1 \pm 3.4$ & $18.8 \pm 1.1$ & $18.7 \pm 1.5$ \\
\hline & & $19.0(17.0-19.0)$ & $19.0(18.0-20.0)$ & $19.0(18.0-20.0)$ & $19.0(18.0-20.0)$ \\
\hline & $65-69$ & $17.9 \pm 2.1$ & $18.3 \pm 1.6$ & $18.0 \pm 2.5$ & $18.2 \pm 1.7$ \\
\hline & & $19.0(16.5-19.0)$ & $19.0(17.5-19.5)$ & $18.0(17.0-19.0)$ & $19.0(17.0-20.0)$ \\
\hline & $70-74$ & $17.1 \pm 2.2$ & $18.1 \pm 2.4$ & $17.8 \pm 1.6$ & $18.0 \pm 2.4$ \\
\hline & & $17.0(16.0-19.0)$ & $19.0(16.0-20.0)$ & $18.0(17.0-19.0)$ & $18.0(17.0-20.0)$ \\
\hline & $75-79$ & $17.3 \pm 2.3$ & $17.8 \pm 2.0$ & $17.0 \pm 1.9$ & $17.9 \pm 2.0$ \\
\hline & & $18.0(16.0-19.0)$ & $18.0(17.0-19.0)$ & $18.0(15.5-18.0)$ & $19.0(17.0-19.0)$ \\
\hline & $\geq 80$ & $16.8 \pm 3.4$ & $16.9 \pm 1.9$ & $16.9 \pm 2.2$ & $17.7 \pm 1.8$ \\
\hline & & $17.0(16.0-19.0)$ & $16.5(15.3-19.0)$ & $17.0(15.5-19.0)$ & $18.0(16.8-19.0)$ \\
\hline \multirow[t]{10}{*}{ Animal fluency } & $60-64$ & $10.8 \pm 2.9$ & $14.6 \pm 3.3$ & $15.2 \pm 3.5$ & $17.6 \pm 4.9$ \\
\hline & & $12.0(9.0-12.0)$ & $14.0(13.3-15.8)$ & $16.0(13.0-18.0)$ & $17.0(15.0-20.5)$ \\
\hline & $65-69$ & $13.2 \pm 3.3$ & $13.9 \pm 3.2$ & $14.1 \pm 3.9$ & $14.3 \pm 0.9$ \\
\hline & & $14.0(10.5-15.5)$ & $14.0(11.0-16.5)$ & $15.0(11.0-17.0)$ & $15.0(14.0-15.0)$ \\
\hline & $70-74$ & $13.0 \pm 3.8$ & $12.9 \pm 3.7$ & $14.1 \pm 3.5$ & $16.8 \pm 5.5$ \\
\hline & & $12.0(10.0-16.0)$ & $12.0(10.0-15.0)$ & $14.0(11.0-16.0)$ & $17.0(13.0-20.0)$ \\
\hline & $75-79$ & $11.6 \pm 4.7$ & $13.8 \pm 3.9$ & $12.6 \pm 4.3$ & $15.5 \pm 4.9$ \\
\hline & & $11.0(9.0-13.0)$ & $13.0(11.0-15.0)$ & $12.0(10.0-15.5)$ & $14.0(12.0-20.0)$ \\
\hline & $\geq 80$ & $11.6 \pm 3.6$ & $12.0 \pm 4.0$ & $11.6 \pm 3.4$ & $13.7 \pm 4.0$ \\
\hline & & $12.0(9.0-14.0)$ & $11.5(9.3-14.0)$ & $11.0(9.0-13.5)$ & $13.0(11.0-16.0)$ \\
\hline \multirow{8}{*}{$\begin{array}{l}\text { Color and object } \\
\text { recognition test }\end{array}$} & $60-64$ & $13.2 \pm 1.8$ & $12.9 \pm 1.5$ & $13.8 \pm 1.5$ & $13.8 \pm 1.3$ \\
\hline & & $13.0(12.0-15.0)$ & $13.0(12.0-14.0)$ & $14.0(13.0-15.0)$ & $14.0(13.0-15.0)$ \\
\hline & $65-69$ & $12.6 \pm 2.1$ & $13.3 \pm 1.7$ & $13.2 \pm 1.5$ & $13.5 \pm 1.4$ \\
\hline & & $13.0(10.0-14.5)$ & $13.0(12.5-15.0)$ & $13.0(12.0-15.0)$ & $14.0(13.0-15.0)$ \\
\hline & $70-74$ & $12.0 \pm 1.8$ & $13.1 \pm 1.7$ & $12.8 \pm 1.6$ & $13.2 \pm 1.8$ \\
\hline & & $12.0(1.0-13.0)$ & $13.0(12.0-14.0)$ & $13.0(12.0-14.0)$ & $14.0(12.0-14.0)$ \\
\hline & $75-79$ & $12.3 \pm 1.8$ & $12.9 \pm 2.0$ & $12.3 \pm 1.6$ & $12.8 \pm 1.8$ \\
\hline & & $13.0(11.0-14.0)$ & $13.0(12.0-14.0)$ & $13.0(11.0-13.5)$ & $13.0(11.0-15.0)$ \\
\hline
\end{tabular}


Table 4. LICA normative data according to age, literacy and education level (continued)

\begin{tabular}{|c|c|c|c|c|c|}
\hline \multirow{2}{*}{ Test } & \multirow{2}{*}{ Age } & \multirow{2}{*}{ Illiteracy } & \multicolumn{3}{|c|}{ Education (years) } \\
\hline & & & $0-3$ & $4-6$ & $\geq 7$ \\
\hline & $\geq 80$ & $10.7 \pm 1.7$ & $12.2 \pm 1.8$ & $11.8 \pm 2.0$ & $12.9 \pm 1.9$ \\
\hline & & $10.0(9.8-12.0)$ & $12.5(11.3-14.0)$ & $12.0(11.0-13.0)$ & $13.0(11.0-15.0)$ \\
\hline \multirow[t]{10}{*}{ Naming } & $60-64$ & $14.2 \pm 0.9$ & $14.0 \pm 1.0$ & $14.4 \pm 0.9$ & $14.4 \pm 0.8$ \\
\hline & & $14.0(13.0-15.0)$ & $14.0(13.0-15.0)$ & $15.0(14.0-15.0)$ & $15.0(14.0-15.0)$ \\
\hline & $65-69$ & $13.8 \pm 1.3$ & $14.2 \pm 1.1$ & $13.7 \pm 1.3$ & $14.3 \pm 0.9$ \\
\hline & & $14.0(13.0-15.0)$ & $15.0(14.0-15.0)$ & $14.0(13.0-15.0)$ & $15.0(14.0-15.0)$ \\
\hline & $70-74$ & $13.2 \pm 1.6$ & $13.9 \pm 1.3$ & $13.6 \pm 1.5$ & $13.7 \pm 1.3$ \\
\hline & & $13.0(12.0-15.0)$ & $14.0(13.0-15.0)$ & $14.0(13.0-15.0)$ & $14.0(13.0-15.0)$ \\
\hline & $75-79$ & $12.9 \pm 1.9$ & $13.5 \pm 1.5$ & $13.5 \pm 1.5$ & $13.6 \pm 1.4$ \\
\hline & & $13.0(12.0-14.0)$ & $14.0(12.0-15.0)$ & $14.0(13.0-15.0)$ & $14.0(13.0-15.0)$ \\
\hline & $\geq 80$ & $11.4 \pm 3.1$ & $13.5 \pm 1.5$ & $12.9 \pm 1.8$ & $13.2 \pm 1.9$ \\
\hline & & $12.5(9.0-13.0)$ & $14.0(13.0-15.0)$ & $13.0(12.0-14.0)$ & $14.0(12.0-15.0)$ \\
\hline \multirow[t]{10}{*}{ LICA total score } & $60-64$ & $198.8 \pm 20.1$ & $222.9 \pm 21.5$ & $226.2 \pm 14.7$ & $235.0 \pm 21.9$ \\
\hline & & $205.6(179.8-215.8)$ & $215.7(211.5-243.8)$ & $225.5(219.0-235.7)$ & $231.5(220.8-251.0)$ \\
\hline & $65-69$ & $206.2 \pm 21.1$ & $218.5 \pm 22.6$ & $219.1 \pm 18.0$ & $228.9 \pm 19.4$ \\
\hline & & $203.3(195.1-224.6)$ & $223.7(205.2-234.0)$ & $220.5(207.0-233.0)$ & $231.2(214.1-241.8)$ \\
\hline & $70-74$ & $194.7 \pm 22.3$ & $212.5 \pm 17.5$ & $215.0 \pm 17.1$ & $222.5 \pm 20.4$ \\
\hline & & $177.0(193.8-211.6)$ & $212.2(202.5-225.4)$ & $218.3(202.0-227.0)$ & $219.8(207.6-241.2)$ \\
\hline & $75-79$ & $192.3 \pm 26.8$ & $208.3 \pm 22.4$ & $201.8 \pm 18.5$ & $218.2 \pm 22.6$ \\
\hline & & $198.5(171.8-213.0)$ & $211.0(201.7-220.2)$ & $202.8(187.0-215.9)$ & $217.3(200.5-235.7)$ \\
\hline & $\geq 80$ & $176.6 \pm 25.0$ & $196.9 \pm 18.2$ & $197.2 \pm 22.5$ & $211.9 \pm 19.9$ \\
\hline & & $182.0(159.0-191.7)$ & $193.3(184.8-210.3)$ & $194.2(184.0-210.0)$ & $209.0(195.7-228.8)$ \\
\hline \multirow[t]{10}{*}{ MMSE } & $60-64$ & $21.1 \pm 4.2$ & $25.5 \pm 2.5$ & $26.4 \pm 2.7$ & $28.1 \pm 1.6$ \\
\hline & & $21.0(18.0-25.0)$ & $25.0(23.3-27.0)$ & $27.0(25.0-29.0)$ & $28.0(27.0-29.0)$ \\
\hline & $65-69$ & $22.6 \pm 2.7$ & $25.9 \pm 2.7$ & $26.5 \pm 2.1$ & $27.7 \pm 1.6$ \\
\hline & & $23.0(20.0-25.0)$ & $26.0(23.5-28.0)$ & $27.0(25.0-28.0)$ & $28.0(27.0-29.0)$ \\
\hline & $70-74$ & $21.8 \pm 3.1$ & $24.9 \pm 2.3$ & $26.1 \pm 2.2$ & $27.3 \pm 1.7$ \\
\hline & & $21.0(20.0-25.0)$ & $25.0(24.0-26.0)$ & $26.0(25.0-27.0)$ & $28.0(26.0-29.0)$ \\
\hline & $75-79$ & $21.1 \pm 3.1$ & $24.0 \pm 3.4$ & $25.9 \pm 2.0$ & $27.4 \pm 1.7$ \\
\hline & & $22.0(19.0-23.0)$ & $24.0(22.0-27.0)$ & $27.0(25.0-27.0)$ & $28.0(26.0-29.0)$ \\
\hline & $\geq 80$ & $19.8 \pm 2.7$ & $23.3 \pm 3.6$ & $25.2 \pm 2.5$ & $26.8 \pm 2.6$ \\
\hline & & $20.0(17.8-22.0)$ & $24.0(22.0-26.0)$ & $25.0(23.0-27.0)$ & $27.0(26.0-28.3)$ \\
\hline
\end{tabular}

${ }^{*}$ mean \pm SD and $t$ median (25-75th percentile). LICA: literacy independent cognitive assessment, MMSE: Mini Mental State Examination

level since it was developed and validated as an effective neurocognitive test battery for illiterate people, ${ }^{11}$ the regression analysis in this study revealed that all of the subtests were significantly affected by education level. This shows that the LICA, a neuropsychological test battery that was developed especially for the illiterate group, is also affected by a subject's education level, which is an intrinsic attribute of any cognitive test. However, regardless of the finding that the LICA test performance was influenced by educational level, it remains the only currently available neuropsychological test battery for illiterate people.

When performing dementia evaluations of illiterate people, it is helpful to interview subjects and their family members using the IADL in order to obtain complimentary information. The IQCODE is particularly useful since it is not affected by the education level or the premorbid ability of subjects. ${ }^{27}$ However, since its data are obtained from the subjects' family members and is not a neuropsychological test, it is difficult to make an accurate assessment of a subject's cognitive function and the result is affected by informant characteristics such as the presence of depression or anxiety, and also by the relationship between the informant and the subject. ${ }^{28}$ Combining neuropsychological testing and the IADL thus likely constitutes a more accurate method of diagnosing dementia.

Literacy is associated with all neuropsychological measures, although the actual correlation between neuropsychological 
test scores and education level depends upon the specific test. Education and learning reinforce and modify certain fundamental abilities, such as verbal/visual memory and visuospatial/visuomotor skills. ${ }^{17}$ Some functional imaging studies have demonstrated that literacy influences the brain and that neural networks for problem-solving and literacy not only impact the individual's daily strategies and function, but also their brain networks. ${ }^{29,30}$ Based on such findings, literacy can be considered to substantially affect neurocognitive dysfunctions such as dementia.

In conclusion, age and literacy/education level substantially influence all the cognitive functions that are assessed by the LICA neuropsychological battery; however, this battery is the only currently specific and available neuropsychological diagnostic method for the evaluation of dementia in illiterate people. The normative data reported herein will be useful for the clinical application of the LICA in illiterate and literate elderly Koreans. Similar normative studies and validations of this tool in different ethnic groups will help to enhance the dementia diagnosis of illiterate people of different ethnicities.

\section{Acknowledgments}

This study was supported by a grant (No. 11-1351000-000049-01) from the Ministry of Health and Welfare, Republic of Korea. This work was supported by the Gachon University Gil Medical Center (Grant number: 2013-43)

\section{REFERENCES}

1. Kinsella KG Velkoff VA. An Aging World: 2001. Washington, DC: Government Printing Office; 2001.

2. Korea National Statistical Office. Statistics for the Elderly 2012. Daejeon: Statistics Korea; 2012.

3. Manly JJ, Jacobs DM, Sano M, Bell K, Merchant CA, Small SA, et al. Effect of literacy on neuropsychological test performance in nondemented, education-matched elders. J Int Neuropsychol Soc 1999;5:191-202.

4. UNESCO. Literacy. Available at: http://portal.unesco.org/education/en/

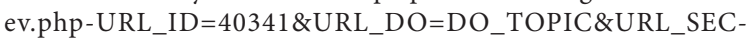
TION=201.html. Accessed January 2014.

5. UNESCO. EFA Global Monitoring Report on Education for All 2006. Paris: Graphoprint; 2005.

6. Byun JI, Lee GA, Huh J, Kim KH. Development Process and Outcomes of Adult Literacy Education in Korea. Seoul: Ministry of Education, Science and Technology; 2012.

7. The National Institute of the Korean Language. The National Literacy Survey. Seoul: The National Institute of the Korean Language; 2008.

8. Lee JY, Chang SM, Jang HS, Chang JS, Suh GH, Jung HY, et al. Illiteracy and the incidence of Alzheimer's disease in the Yonchon County survey, Korea. Int Psychogeriatr 2008;20:976-985.

9. Fuh JL, Teng EL, Lin KN, Larson EB, Wang SJ, Liu CY, et al. The Informant Questionnaire on Cognitive Decline in the Elderly (IQCODE) as a screening tool for dementia for a predominantly illiterate Chinese population. Neurology 1995;45:92-96.

10. Jorm A, Scott R, Cullen J, MacKinnon AJ. Performance of the Informant Questionnaire on Cognitive Decline in the Elderly (IQCODE) as a screening test for dementia. Psychol Med 1991;21:785-790.

11. Choi SH, Shim YS, Ryu SH, Ryu HJ, Lee DW, Lee JY, et al. Validation of the Literacy Independent Cognitive Assessment. Int Psychogeriatr 2011; 23:593-601.

12. Kim J, Jeong JH, Han SH, Ryu HJ, Lee JY, Ryu SH, et al. Reliability and validity of the short form of the literacy-independent cognitive assessment in the elderly. J Clin Neurol 2013;9:111-117.

13. Christensen KJ, Multhaup KS, Nordstrom S, Voss K. A cognitive battery for dementia: development and measurement characteristics. J Consult Clin Psychol 1991;3:168-174.

14. Kang Y. Normative data on the Korean Mini-Mental State Examination (K-MMSE) in Korean elderly. Kor J Psychol 2006;25:1-25.

15. Kang SJ, Choi SH, Lee BH, Kwon JC, Na DL, Han SH. The reliability and validity of the Korean Instrumental Activities of Daily Living (KIADL). J Korean Neurol Assoc 2002;20:8-14

16. Ardila A, Rosselli M, Rosas P. Neuropsychological assessment in illiterates: visuospatial and memory abilities. Brain Cogn 1989;11:147-166.

17. Ardila A, Ostrosky-Solis F, Mendoza VU. Learning to read is much more than learning to read: a neuropsychologically based reading program. J Int Neuropsychol Soc 2000;6:789-801.

18. Bertolucci PH, Brucki SM, Campacci SR, Juliano Y. The Mini-Mental State Examination in a general population: impact of educational status. Arq Neuropsiquiatr 1994;52:1-7.

19. Brucki SM, Rocha MS. Category fluency test: effects of age, gender and education on total scores, clustering and switching in Brazilian Portuguese-speaking subjects. Braz J Med Biol Res 2004;37:1771-1777.

20. Dansilio S, Charamelo A. Constructional functions and figure copying in illiterates or low-schooled Hispanics. Arch Clin Neuropsychol 2005; 20:1105-1112.

21. Deloche G, Souza L, Braga LW, Dellatolas G. A calculation and number processing battery for clinical application in illiterates and semi-literates. Cortex 1999;35:503-521.

22. Folia V, Kosmidis MH. Assessment of memory skills in illiterates: strategy differences or test artifact? Clin Neuropsychol 2003;17:143-152.

23. Kosmidis MH, Tsapkini K, Folia V, Vlahou CH, Kiosseoglou G. Semantic and phonological processing in illiteracy. J Int Neuropsychol Soc 2004; 10:818-827.

24. Nitrini R, Caramelli P, Herrera Junior E, Porto CS, Charchat-Fichman $\mathrm{H}$, Carthery MT, et al. Performance of illiterate and literate nondemented elderly subjects in two tests of long-term memory. J Int Neuropsychol Soc 2004;10:634-638.

25. Nitrini R, Caramelli P, Herrera E Jr, Charchat-Fichman H, Porto CS. Performance in Luria's fist-edge-palm test according to educational level. Cogn Behav Neurol 2005;18:211-214.

26. Lee DY, Lee KU, Lee JH, Kim KW, Jhoo JH, Kim SY, et al. A normative study of the CERAD neuropsychological assessment battery in the Korean elderly. J Int Neuropsychol Soc 2004;10:72-81.

27. Perroco TR, Bustamante SE, Moreno Mdel P, Hototian SR, Lopes MA, Azevedo D, et al. Performance of Brazilian long and short IQCODE on the screening of dementia in elderly people with low education. Int Psychogeriatr 2009;21:531-538.

28. Jorm AF. The Informant Questionnaire on cognitive decline in the elderly (IQCODE): a review. Int Psychogeriatr 2004;16:275-293.

29. Ardila A, Bertolucci PH, Braga LW, Castro-Caldas A, Judd T, Kosmidis $\mathrm{MH}$, et al. Illiteracy: the neuropsychology of cognition without reading. Arch Clin Neuropsychol 2010;25:689-712.

30. Aprahamian I, Martinelli JE, Cecato J, Yassuda MS. Screening for Alzheimer's disease among illiterate elderly: accuracy analysis for multiple instruments. J Alzheimers Dis 2011;26:221-229. 